\title{
Recomendaciones contextuales basadas en el enfoque de post-filtrado
}

\author{
Xochilt Ramírez-García, Mario García-Valdéz \\ Instituto Tecnológico de Tijuana, Tijuana, B.C., \\ México \\ xochilt.ramirez@gmail.com, mariosky@gmail.com
}

\begin{abstract}
Resumen. Hoy en día, los sistemas de recomendación son utilizados en diferentes tipos de aplicaciones. Un sistema de recomendación de restaurantes proporciona recomendaciones considerando los gustos y preferencias del usuario con el propósito de facilitar al usuario la selección de restaurantes que posteriormente visitará. El objetivo de este trabajo es desarrollar una aplicación para hacer recomendaciones de restaurantes que permitan difundir información sobre productos y servicios ofrecidos en Tijuana a través de una página Web. El algoritmo de recomendación propuesto se basa en el enfoque de post-filtrado, utiliza el algoritmo de filtrado colaborativo y la información contextual de la situación actual del usuario para obtener recomendaciones contextuales. El conjunto de datos fue adquirido a través de cuestionarios contestados por 50 personas, se hicieron recomendaciones con una matriz de valoraciones que contiene 1,422 votos contextuales, 50 usuarios y 40 restaurantes. Se utilizó el Error Absoluto Medio para evaluar el desempeño del sistema de recomendación.
\end{abstract}

Palabras clave: Sistemas de recomendación, filtrado colaborativo, contexto, post-filtrado.

\section{Introducción}

Los seres humanos tienen un proceso de comunicación para transmitir ideas y reaccionan apropiadamente, esto se debe a factores como la riqueza del lenguaje y la comprensión de las palabras en diferentes situaciones. Cuando las personas conversan usan implícitamente información situacional (o contextual) logrando aumentar el rango de la conversación. Utilizando la información contextual se incrementa la comunicación en la interacción humano-computadora, esto contribuye a mejorar el desarrollo de aplicaciones inteligentes. Este proceso también es aplicado en los sistemas de recomendación sensibles al contexto.

Actualmente los sistemas de recomendación pueden ser usados en aplicaciones modernas que muestran al usuario una amplia variedad de ítems. Tales sistemas, generalmente proporcionan al usuario una lista de ítems que prefiere o predicen el 
grado de cuánto podría gustarle un cierto ítem al usuario, el objetivo es ayudarle a seleccionar un ítem dentro una gran cantidad de estos.

La mayoría de sistemas de recomendación se centran en los ítems más relevantes para el usuario y no consideran información contextual como tiempo, lugar o acompañantes, por ejemplo para ver una película [9] o salir a cenar [22]. En otras palabras, los sistemas de recomendación trabajan con las aplicaciones considerando solo dos tipos de entidades: usuarios e ítems, y no utilizan el contexto cuando proveen recomendaciones al usuario.

En aplicaciones como recomendación de paquetes turísticos [6, 7], recomendación de contenidos personalizados en una página Web [18], o recomendación de una película [16], puede ser insuficiente solo considerar información acerca de los usuarios e ítems, es importante considerar información contextual en el proceso de recomendación con el fin de sugerir ciertos ítems en ciertas circunstancias [28]. Recientemente, existe una gran cantidad de investigaciones en el área de sistemas de recomendación y la mayoría se enfoca en diseñar algoritmos para recomendaciones contextuales $[6,19,15]$. Esta investigación propone el uso del enfoque de postfiltrado que utiliza el algoritmo de filtrado colaborativo y la información contextual del usuario en el dominio de restaurantes.

Los temas en el trabajo se presentan como sigue: en la sección 2, se describen los conceptos relacionados; en la sección 3, se explica la arquitectura del sistema de recomendación propuesto; en la sección 4 , se define la configuración del ambiente, el conjunto de datos obtenido, las métricas y los experimentos para evaluar el algoritmo; en la sección 5, se explican los resultados de la evaluación; y en la sección 6, se explican las conclusiones del estudio, los puntos más importantes del enfoque, las lecciones aprendidas y el trabajo futuro.

\section{Sistemas de recomendación}

Los sistemas de recomendación forman parte de la solución al problema de la sobrecarga de información que sufren los consumidores en Internet. Estos sistemas han sido probados para ser una de las técnicas más utilizadas que ayudan a los usuarios a encontrar ítems relevantes para ellos [2].

\subsection{Algoritmo de filtrado colaborativo}

Un algoritmo de filtrado colaborativo [2] hace recomendaciones analizando la correlación entre usuarios. El algoritmo calcula la similaridad entre los usuarios para hacer predicciones. Por ejemplo, suponiendo que el usuario A es similar al usuario B, si el usuario A esta interesado en el producto $X$, entonces se puede inferir que el usuario $\mathrm{B}$ también estará interesado en el producto $\mathrm{X}$, entonces el producto $\mathrm{X}$ será recomendado al usuario $\mathrm{B}$. El enfoque de filtrado colaborativo necesita recolectar información de los votos de los usuarios sobre los ítems, e identificar vecindarios tomando en cuenta la similaridad entre estos. Este enfoque se basa en el supuesto de 
que cuando las personas seleccionan productos o información son influenciadas por las opiniones de otras personas que han tenido alguna experiencia con el producto o la información. Además, en el filtrado colaborativo, el análisis del contenido de los ítems es ignorado y solo usa las opiniones sobre los ítems que son considerados más relevantes. Esto es importante para recomendar ítems para los cuales el análisis de contenido es escaso [26].

\subsection{Contexto}

En [4] definen el contexto como el estado físico, social, emocional o formativo en el del usuario. El contexto es toda la información acerca de la situación pertinente a una aplicación y su conjunto de usuarios, aunque en ocasiones, no toda la información es considerada relevante para la aplicación. Es imposible enumerar cuáles son los aspectos importantes de todas las situaciones, ya que esto cambia de una situación a otra, por ejemplo, en algunos casos el entorno físico puede ser importante mientras que en otros puede ser completamente irrelevante.

Definición. El contexto es cualquier información que se puede utilizar para caracterizar la situación de una entidad. Una entidad es una persona, lugar u objeto que se considera relevante para la interacción entre un usuario y una aplicación, incluyendo el usuario y aplicaciones en sí [4].

La definición anterior facilita la enumeración de los aspectos del contexto de una aplicación dada. Si una pieza de información puede ser utilizada para caracterizar la situación de un participante en una interacción, entonces esta información será el contexto [13].

\subsection{Enfoque de post-filtrado}

El enfoque post-filtrado inicialmente ignora la información contextual en la primera etapa de las recomendaciones, es decir, cuando se genera la lista ordenada de los $\mathrm{N}$ ítems seleccionados para la lista de recomendaciones. Posteriormente, el enfoque post-filtrado ajusta la lista de recomendaciones para cada usuario utilizando la información contextual. Los ajustes de la lista de recomendación pueden ser hechas de dos maneras [3]:

- Filtrando las recomendaciones que son irrelevantes (en un contexto dado), o

- Ajustando la clasificación de las recomendaciones de la lista (en base al contexto dado).

En la figura 1 se representa el esquema del proceso de post-filtrado contextual. En este proceso se analizan los datos de preferencia de contexto para un usuario determinado en un contexto dado para encontrar los patrones de uso específicos de los ítems y luego usar estos patrones para ajustar la lista de elementos, dando lugar a las recomendaciones contextuales. En otras palabras, el post-filtrado desecha los ítems recomendados que son menos relevantes en el contexto específico y mantiene los elementos más relevantes. La ventaja más importante del post-filtrado contextual es 
que permite el uso de cualquiera de las numerosas técnicas de recomendación tradicionales previamente propuestas en la literatura [2].

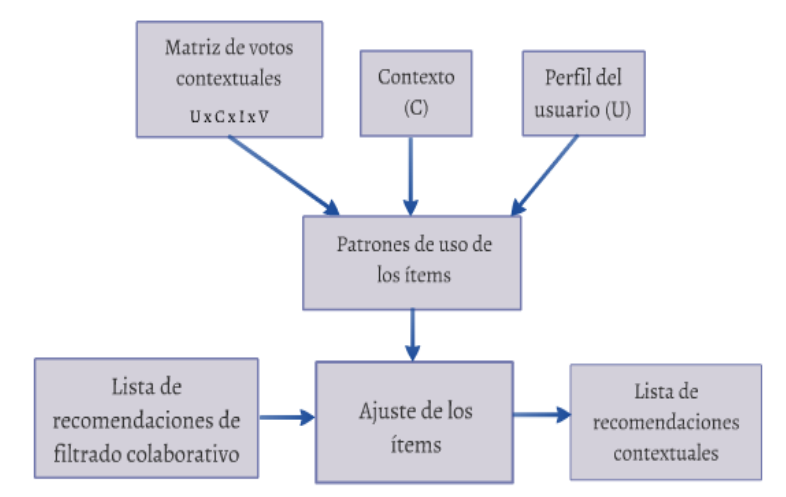

Fig. 1. Esquema del proceso de post-filtrado para obtener recomendaciones contextuales [3].

\section{Sistema de recomendación de restaurantes}

El sistema de recomendación de restaurantes utiliza el algoritmo de filtrado colaborativo para encontrar restaurantes relevantes para el usuario [22]. Las votaciones en el perfil de usuario (representado como un vector de enteros) son utilizadas para determinar la similaridad entre estos, es decir, qué tan parecidos son unos con otros considerando el perfil de cada usuario. El vecindario para el usuario es obtenido del conjunto de usuarios con mayor similaridad. La similaridad es calculada con la correlación de Pearson. Posteriormente, el algoritmo obtiene una lista de los 10 mejores restaurantes recomendados para el usuario. La salida del algoritmo de filtrado colaborativo suministra la siguiente etapa de post-filtrado y en la lista de recomendaciones el algoritmo agrega el promedio de los votos para cada ítem de la lista.

La etapa de filtrado de restaurantes por contexto consiste clasificar todos los votos en el contexto que corresponde, es decir, de acuerdo a la fecha en que fueron votados el sistema los clasifica en el contexto "entre semana" o "fin de semana".

El contexto actual es la información acerca del momento en que el usuario esta interactuando con el sistema. En la etapa de selección de restaurantes en el contexto actual, el sistema analiza cuáles son los restaurantes votados en el mismo contexto del usuario actual y guarda esos datos para empezar a compararlos con los ítems que están en el mismo contexto, finalmente obtiene una lista de ítems recomendados contextualizada.

En la etapa del cálculo de la predicción por promedio, el sistema obtiene el promedio de los votos que están en el mismo contexto para cada ítem de la lista contextualizada. Enseguida, para calcular la predicción por promedio, se utiliza: 1) la predicción del algoritmo de filtrado colaborativo del ítem y, 2) el promedio del ítem en el contexto actual, es decir, el promedio de las votaciones de los usuarios 
previamente calculado. La lista de recomendaciones contextuales obtenida en la última etapa contiene los restaurantes con altas predicciones (basada en el umbral de 3.5). El esquema del proceso se representa en la figura 2.

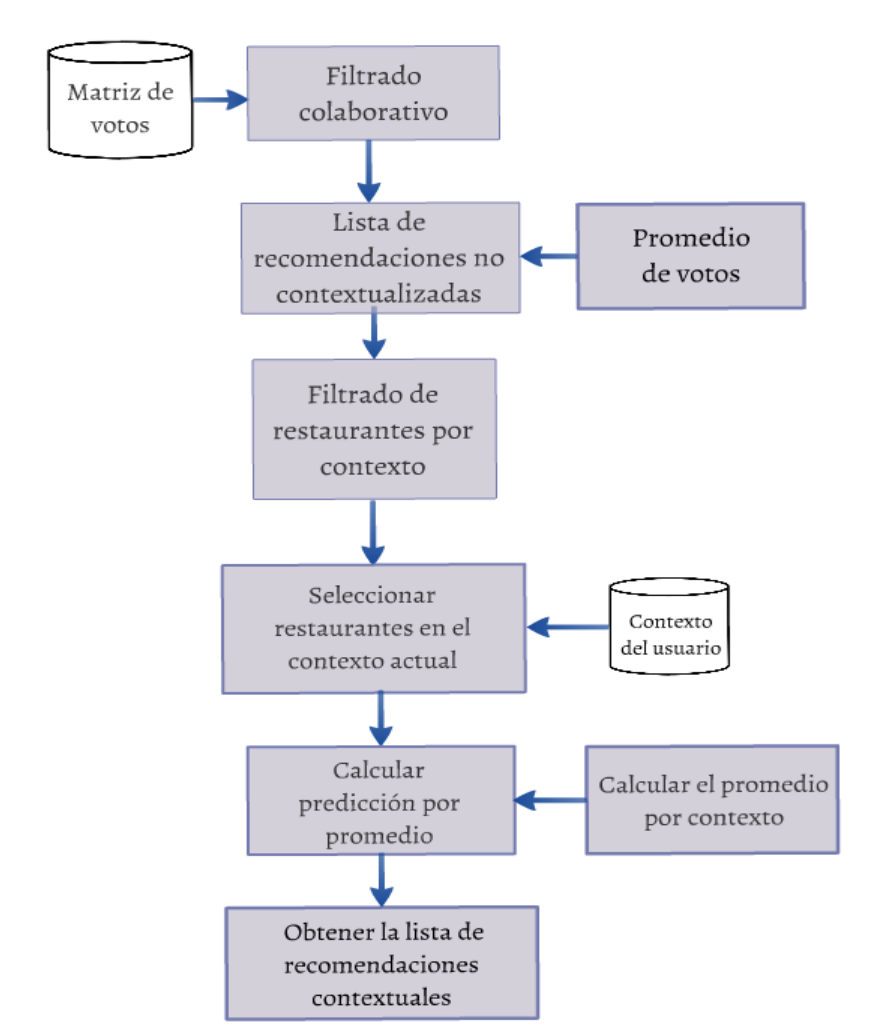

Fig. 2. Metodología de post-filtrado en el sistema de recomendación de restaurantes.

\section{Configuración}

\subsection{Conjunto de datos}

Para recolectar el conjunto de datos se aplicó un cuestionario a los estudiantes de Ingeniería en Sistemas, estudiantes de Maestría en Ciencias Computacionales y profesores del Instituto Tecnológico de Tijuana. Un total de 50 personas respondieron el cuestionario, las preguntas solicitaban información principalmente acerca de sus gustos y preferencias sobre los restaurantes cercanos al Instituto Tecnológico de Tijuana y la tecnología o recursos utilizados para buscar opciones de restaurantes. El cuestionario consta de 8 preguntas donde las personas, asignan votos a los restaurantes que han visitado de una lista de 40. Cada restaurante elegido fue calificado 6 veces, una para cada contexto, sin embargo, hubo quienes votaban menos contextos y quienes no votaban ningún restaurante. Al final de la encuesta se 
obtuvieron un total de 1,422 votos. Las preguntas del cuestionario se enlistan en la tabla 1 al igual que las posibles respuestas.

Tabla 1. Cuestionario para obtener la retroalimentación del usuario.

\begin{tabular}{|c|c|c|}
\hline Pregunta & \multicolumn{2}{|c|}{ Respuesta } \\
\hline 1. ¿Cuál es tu ocupación? & 1. Estudiante & 2.Empleado \\
\hline $\begin{array}{l}\text { 2. De acuerdo a su prioridad, ordene } \\
\text { por importancia las características } \\
\text { que consideras cuando escoges un } \\
\text { restaurante. }\end{array}$ & $\begin{array}{l}\text { 1.Instalacion } \\
\text { 2. Precio } \\
\text { 3. Servicios }\end{array}$ & $\begin{array}{l}\text { 4. Platillos } \\
\text { 5. Ambiente } \\
\text { 6. Ubicación }\end{array}$ \\
\hline $\begin{array}{l}\text { 3. ¿Qué tecnología utilizas con más } \\
\text { frecuencia? }\end{array}$ & $\begin{array}{l}\text { 1. Teléfono } \\
\text { inteligente } \\
\text { 2. Tableta }\end{array}$ & $\begin{array}{l}\text { 3. Laptop } \\
\text { 4. PC }\end{array}$ \\
\hline $\begin{array}{l}\text { 4. ¿Qué sistemas operativos } \\
\text { utilizas? }\end{array}$ & $\begin{array}{l}\text { 1. Android } \\
\text { 2. Windows } \\
\text { 3. iOS }\end{array}$ & $\begin{array}{l}\text { 4. Symbian } \\
\text { 5.BlackberryOS } \\
\text { 6. Otro }\end{array}$ \\
\hline $\begin{array}{l}\text { 5. ¿Has utilizado alguna aplicación } \\
\text { para buscar restaurantes en Tijuana? }\end{array}$ & $\begin{array}{l}\text { 1. Si } \\
\text { 2. No }\end{array}$ & 3. ¿Cuál? \\
\hline $\begin{array}{l}\text { 6. ¿Te gustaría usar una aplicación } \\
\text { de recomendación de restaurantes de } \\
\text { Tijuana? }\end{array}$ & 1. $\mathrm{Si}$ & 2. No \\
\hline $\begin{array}{l}\text { 7. Asigne una calificación a los } \\
\text { restaurantes que prefieras sin } \\
\text { considerar situaciones contextuales. }\end{array}$ & Lista de Restaurante & de Tijuana \\
\hline $\begin{array}{llr}\text { 8. Asigne una } & \text { calificación a los } \\
\text { restaurantes } & \text { que } & \text { prefieras } \\
\text { considerando } & & \text { situaciones } \\
\text { contextuales. } & & \end{array}$ & Lista de Restaurante & de Tijuana \\
\hline
\end{tabular}

Posteriormente, el total de las respuestas a las preguntas 1-6 se muestran en la Tabla 2. Las respuestas de las preguntas 2, 3 y 4 demuestran las preferencias de los usuarios. La respuesta de la pregunta 2 demuestra que tanta importancia tiene cada uno de los elementos propuestos, es decir, cuál es el factor que determina la toma de decisión al elegir visitar un restaurante. En la tabla 2 está la cantidad de usuarios que prefieren como su favorito cada uno de los factores propuestos. En la respuesta de la pregunta 3, los usuarios revelan cuál es el dispositivo que utilizan comúnmente para buscar opciones de restaurantes. En su mayoría los usuarios utilizan laptops, las prefieren por su portabilidad y fácil acceso a las redes y enseguida los teléfonos celulares inteligentes que proporcionan los mismos servicios y accesos. La respuesta de la pregunta 3, demuestra que Windows y Android son los sistemas operativos utilizan la mayoría, siendo estos los más fáciles de usar y obtener para estudiantes y maestros. Las respuestas de la preguntas 5 y 6 demuestran lo inusual que es el uso de un sistema de recomendación de restaurantes entre los estudiantes y maestros, siendo esta una motivación más para desarrollar la aplicación. 
Tabla 2. Total de respuestas de los usuarios obtenidas en el cuestionario.

\begin{tabular}{|c|r|r|r|r|r|r|}
\hline \multirow{2}{*}{ Pregunta } & \multicolumn{7}{|c|}{ Respuestas } \\
\cline { 2 - 7 } & 1 & 2 & 3 & 4 & 5 & 6 \\
\hline 1 & 4 & 46 & & & & \\
\hline 2 & 6 & 9 & 8 & 13 & 2 & 10 \\
\hline 3 & 30 & 8 & 35 & 17 & & \\
\hline 4 & 38 & 39 & 20 & 4 & 6 & 5 \\
\hline 5 & 6 & 42 & & & & \\
\hline 6 & 46 & 3 & & & & \\
\hline
\end{tabular}

La respuesta a las preguntas 7 y 8 se representan con las graficas de la figura 3 , donde se observa la cantidad de votos obtenidos para cada restaurante, siendo estos los 10 restaurantes favoritos de los usuarios sin considerar información contextual (figura a) y los 10 restaurantes favoritos considerando información contextual (figura b).
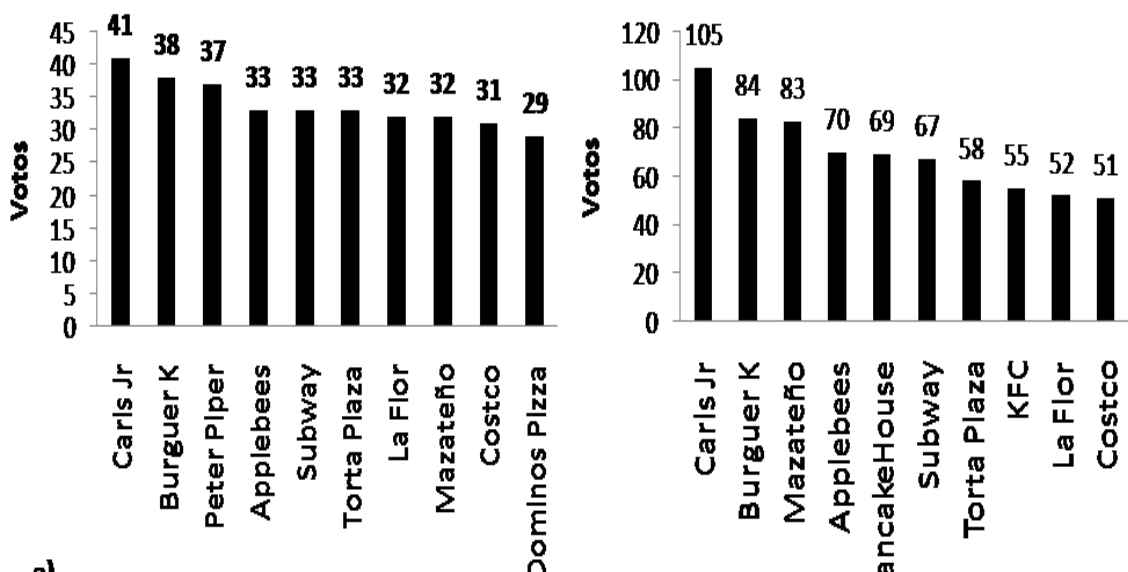

a)

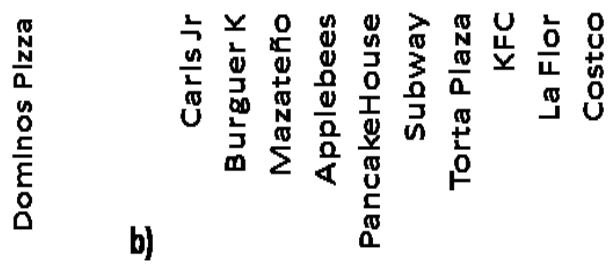

Fig. 3. Gráficas de las preferencias de los usuarios sobre los restaurantes propuestos en el sistema de recomendación.

En la gráfica a), los restaurantes favoritos de acuerdo a los votos de los usuarios fueron "Carls Jr" y "Burguer King" con mayor cantidad de votos, en la gráfica b), se aprecia la diferencia a partir del tercer restaurante, donde los restaurantes cambian de posición o simplemente no aparecen en alguna lista. Las recomendaciones contextuales del enfoque de post-filtrado depende del factor contextual "Día de la semana", que significa el día en que se votaron los restaurantes.

Posteriormente, el resultado de la consulta es refinada de acuerdo al contexto del usuario actual; los 6 contextos mencionados que corresponden a combinaciones de factores contextuales se describen en la Tabla 3. 
Xochilt Ramírez-García, Mario García-Valdéz.

Tabla 3. Factores contextuales considerados en el post-filtrado.

\begin{tabular}{|l|l|}
\hline Factor contextual & \multicolumn{1}{|c|}{ Dominio } \\
\hline Día de la semana & $\begin{array}{l}\text { Entre semana (lunes, martes, miércoles y jueves) } \\
- \text { Fin de semana (viernes, sábado y domingo) }\end{array}$ \\
\hline Lugar & $\begin{array}{l}\text { - Escuela } \\
- \text { Casa } \\
\end{array}$ \\
& - Trabajo \\
\hline
\end{tabular}

\subsection{Métricas}

Sistemas de Recomendación son ampliamente utilizados en diferentes dominios, por lo tanto, los objetivos pueden ser diferentes en cada aplicación, las métricas utilizadas dependen del objetivo del sistema. Para algunas aplicaciones la precisión de la predicción es fundamental y la evaluación del sistema se centra en la precisión de las recomendaciones. Existen varios indicadores para medir la precisión: Error Cuadrático Medio (RMSE por sus siglas en inglés) [9, 10], el Error Absoluto Medio (MAE por sus siglas en inglés) [9] y la Precisión y Relevancia [9, 10], son algunos ejemplos. Por otro lado, si el sistema intenta medir aspectos cualitativos como la satisfacción del usuario, calidad de las recomendaciones o utilidad de sistema para un grupo de usuarios o comunidad, se consideran otras métricas como se menciona en [14].

Precisión en la predicción. Precisión de la predicción es la propiedad más discutida en la literatura sistema de recomendación. El centro de la gran mayoría de los sistemas de recomendación son motores de predicción. Este motor puede predecir las opiniones de los usuarios sobre los elementos (por ejemplo, las calificaciones de las películas) [9] o la probabilidad de uso (por ejemplo en compras de consumidores) [24]. Un supuesto básico en los sistemas de recomendación es que el usuario va a preferir un sistema que proporciona predicciones más precisas, a raíz de esto muchos investigadores se han propuesto encontrar algoritmos que proporcionen mejores predicciones. La precisión de la predicción es normalmente independiente de la interfaz del usuario, por lo que se puede medir en un experimento off-line. La medición de precisión en un estudio de usuarios significa medir la exactitud dada una recomendación. Este es un concepto diferente de la predicción del comportamiento de los usuarios sin recomendaciones, y está más cerca de la verdadera exactitud en el sistema real [14].

Error Absoluto Medio (MAE). El Error Absoluto Medio es una métrica popular usada en la evaluación de la precisión de las predicciones, el sistema genera predicciones $\hat{r}_{u i}$ para un conjunto de datos de prueba $\mathscr{T}$ de los pares usuario-item (ui) para el cual el verdadero rating $\boldsymbol{r}_{u i}$ es conocido. Típicamente, $\boldsymbol{r}_{u i}$ es conocido 
porque está oculto en un experimento off-line, o porque fue obtenido a través de un estudio del usuario o experimento on-line [14]. El error entre la predicción y el voto real es dado por la siguiente ecuación (1):

$$
\mathrm{MAE}=\sqrt{\frac{1}{|\mathscr{T}|} \sum_{(u, i) \in \mathscr{T}}\left|\hat{r}_{u i}-r_{u i}\right|}
$$

\section{Resultados del experimento}

El algoritmo fue desarrollado en el lenguaje Python y probado con un gestor de base de datos PostgreSQL, el conjunto de datos se recolectó explícitamente de 50 usuarios que contestaron el cuestionario detallado en la sección 4.1. La figura 5 muestra la distribución de los votos en una matriz tridimensional obtenida con los votos (en una escala de 1 a 5), 40 restaurantes y 6 diferentes situaciones contextuales, en total la matriz que contiene 1,422 votos. Se realizó un total de 172 predicciones para usuarios tomados aleatoriamente y el error fue de 0.58. Las pruebas se hicieron con diferentes usuarios y en diferentes contextos, el error calculado se obtiene de todas las predicciones hechas aleatoriamente. El resultado está limitado al tamaño del conjunto de datos, que en este caso particular restringe el rendimiento del algoritmo. Por otro lado, tener el "día de la semana" como principal factor contextual no mejora la precisión de las recomendaciones en este dominio.

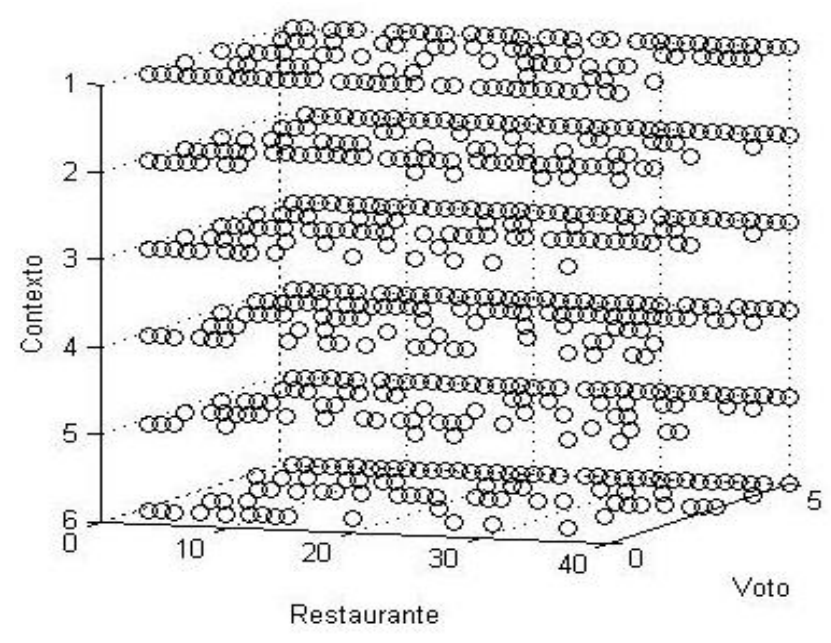

Fig. 4. Distribución de los votos en los 6 contextos propuestos.

En un sistema de recomendación que utiliza algoritmos sin información contextual, las predicciones pueden ser menos precisas con un error de 0.77 , como se ha hecho anteriormente en trabajo previo [22], sin embargo estas predicciones están basadas solamente en el perfil de usuario (usuario-ítem) y no consideran información diferente de los votos (filtrado colaborativo) o diferente a la descripción de los restaurantes 
(basado en contenido). Además el conjunto de datos utilizado en el trabajo previo no era más grande que el que proponemos en este trabajo, el tamaño del conjunto de datos afecta el desempeño del algoritmo.

Al agregar información contextual la complejidad del proceso de recomendación en el post-filtrado aumenta. La matriz de votos contextuales en cualquier caso tendrá al menos 3 dimensiones (usuario-ítem-contexto), cada factor contextual agregado representa una dimensión más en la misma. Por lo tanto, hacer la selección de la información contextual para el usuario es una tarea compleja que aumenta cuando los conjuntos de datos son robustos pero a la vez permite obtener recomendaciones más precisas y apegadas a la realidad del usuario.

\section{Conclusiones y trabajo futuro}

La cantidad de información que se suministra para el sistema es fundamental para lograr recomendaciones más exactas [26]. El problema de la escasez de información contextual [20] conduce a malas recomendaciones y es por lo tanto, un problema latente en este trabajo debido al tamaño del conjunto de datos con poca información contextual. Otro problema encontrado al analizar los resultados del cuestionario, es que los usuarios no cambian realmente sus calificaciones cuando se le preguntó a considerar un contexto particular. Una posible explicación de este comportamiento la carga cognitiva adicional que los usuarios tienen al pensar en una diferencia en la preferencia en cada escenario, es posible que su preferencia pueda ser determinada independientemente del contexto y no es hasta que se enfrenta realmente con el problema de tomar la decisión de elegir un restaurante entre distintos competidores en un contexto particular, cuando los usuarios asignan un rating de utilidad a cada opción elegida.

Los factores contextuales utilizados en otras investigaciones [6], [19], [11] son basados en el dominio del sistema de recomendación, estos factores se pueden incluir desde las consultas a la base de datos y se utilizan como referencia para la clasificación de los ítems [19], en base a las preferencias del usuario [22], o a los modelos de probabilidad para predecir el comportamiento de los usuarios [23].

Sin embargo, en el experimento se identificó que los usuarios tenían opiniones diferentes cuando asumen situaciones contextuales y cuando ocurre la situación en la vida real. La selección de un restaurante podría verse afectada no sólo por los gustos y preferencias de los usuarios, sino también por otros factores que no son percibidos en esas suposiciones. El usuario no toma decisiones teniendo en cuenta los factores contextuales, como los días de la semana, el dispositivo móvil o la distancia, de hecho, sería interesante preguntarse cómo influye la distancia cuando el usuario es estudiante y cómo cambia la decisión cuando el usuario no es un estudiante, o cómo la distancia influye en la decisión cuando el usuario interactúa con diferentes dispositivos. Otros factores (los que podrían ser subjetivos) son cada vez más relevantes: 1) las relaciones personales (amigos, familiares y compañeros de trabajo), 2) el dinero con que cuenta en ese momento, 3) el tipo de alimentos que el usuario prefiere consumir o que se le antoja debido al clima ó a su estado de ánimo, ó 4) 
simplemente por el hábito de ir al mismo lugar. Somos conscientes de que hay una diferencia en la selección del restaurante, los deseos del usuario (lo que quiere el usuario en ese momento) son más importantes que sus preferencias ó gustos, y también es prioritario considerar la influencia del razonamiento lógico para decidir qué restaurante visitará.

Por otra parte, la información contextual obtenida de los usuarios a través del cuestionario no es totalmente fiable, la razón es que la mayoría de los estudiantes no mostraron una diferencia entre visitar un lugar en fin de semana o entre semana, el voto fue el mismo en los diferentes contextos. En el peor de los casos, los estudiantes solo ignoraron la pregunta dejando votos vacíos que el sistema no puede inferir si la información es escasa o nula.

En este estudio se observó que para los usuarios hacer suposiciones al votar fue extenuante [8], esta razón no facilita obtener información relevante, probablemente, las respuestas de los estudiantes no fueron las correctas en comparación con una situación de la vida real donde el usuario antes de seleccionar piensa acerca de los criterios que deben cumplirse para visitar un restaurante. Entonces, el "día de la semana" y "el lugar" no se consideran factores primordiales para estas recomendaciones contextuales, el experimento demostró que para los estudiantes no es importante que la situación contextual cambie.

De acuerdo a las lecciones aprendidas en este estudio, tomando en cuenta el desempeño del algoritmo y con el objetivo de mejorarlo, las propuestas para trabajo futuro son las siguientes:

- El utilizar en el factor contextual "día de la semana" un mayor rango del dominio, que permita al usuario identificar claramente las diferencias cuando el contexto cambia.

- Utilizar la localización geográfica para identificar los puntos donde se encuentra el usuario y las ubicaciones de los restaurantes, esto no limitaría al usuario a consumir en restaurantes cercanos al Instituto Tecnológico de Tijuana.

- Utilizar otras maneras de obtener información relevante del contexto con un esfuerzo mínimo de usuario, puede hacerse de forma implícita, como se menciona en [17].

- Implementar otro enfoque sistema de recomendación (por ejemplo, pre-filtrado) y compararlo con las recomendaciones de post-filtrado, esto con el fin de evaluar la precisión de la lista de recomendaciones en ambos casos.

- Probar y evaluar el algoritmo en un dominio diferente para observar el rendimiento con diferente información contextual.

- Para mejorar los resultados se deben aplicar más pruebas al sistema de recomendación con grandes conjuntos de datos y en otros dominios (por ejemplo, cursos de aprendizaje en línea), donde la información contextual es fundamental para lograr recomendaciones más precisas.

\section{Referencias}

1. Abbar, S., Bouzeghoub, M., Lopez, S.: Context-Aware Recommender Systems: A service oriented approach. In: VLDB PersDB Workshop (2009) 
2. Adomavicius, G.; Tuzhilin, A.: Toward the next generation of recommender systems: a survey of the state-of-the-art and possible extensions. Transactions on Knowledge and Data Engineering, vol. 17, pp. 734-749 (2005)

3. Adomavicius, G., Tuzhilin, A.: Context-Aware Recommender Systems. Recommender Systems Handbook. pp. 217-253 (2011)

4. Dey, A., Abowd, K., Gregory, D.: Towards a better understanding of context and contextawareness. Graphics, Visualization and Usability Center and College of Computing. Georgia Institute of Technology, Atlanta, GA. (1999)

5. Baltrunas, L., Ricci, F.: Context-Based Splitting of Item Ratings in Collaborative Filtering. In: Proceedings of the ACM Cionference Series on Recommender Systems (2009)

6. Baltrunas, L., Ludwig, B., Peer, S., Ricci, F.: Context-Aware Places of Interest Recommendations and Explanations. Free University of Bozen-Bolzano. Bolzano, Italy (2011)

7. Baltrunas, L., Ludwig, B., Peer, S., Ricci, F.: Context relevance assesment and exploitation in mobile recommender systems. Personal and Ubiquitous Computing. Free University of Bolzano. Bolzano, Italy (2011)

8. Baltrunas, L., Ludwig, B., Ricci, F.: Context Relevance Assessment for Recommender Systems. In: Proceedings of the International Conference on Intelligent User Interfaces. pp. 287-290 (2011)

9. Campochiaro, E., Cassata, R., Cremonesi, P., Turrin, R.: Do Metrics Make Recommender Algorithms? In: Proceedings of International Conference on Advanced Information Networking and Applications Workshops. Milano, Italy (2009)

10. Caraciolo, M.: Artificial Intelligence in Motion.

http://aimotion.blogspot.mx/2011/05/evaluating-recommender-systems.html. Fecha de consulta: noviembre del 2013.

11. Chung-hua, C., Se-Hsien, W.: A chinese Restaurant Recommendation System based on Mobile context-Aware Services. In: Proceedings of $14^{\text {th }}$ International Conference on Mobile Data Management. IEEE. National Taichung University of Science and Technology (2013)

12. Jannach, D., Zanker, M., Felfernig, A., Friedrich, G.: Recommender Systems: An Introduction. Cambridge University Press (2010)

13. Fischer, G.: Context-Aware Systems - The Right Information, at the Right Time, in the Right Place, in the Right Way, to the Rigth Person. ACM. University of Colorado, Boulder, USA (2012)

14. Guy, S., Gunawardana, A.: Evaluating Recommendation Systems. Microsoft Research (2009)

15. Hussein, T., Linder, T., Gaulke, W., Ziegler, J.: Context-Aware recommendations on rails. (2009)

16. Kyung-Rog, K., Ju-Ho, L., Jae-Hee, B.: Recommender System Using the Movie Genre similarity in Mobile Service. Proceedings of $4^{\text {th }}$ Internartional Conference on Multimedia and Ubiquitous Engineering (MUE) (2010)

17. Lakshmish, R. et al.: CAESAR: A context-aware, social recommender system for low-end mobile devices. Proceedings of International Conference on mobile Data Management: systems, and services and middleware. IEEE (2009)

18. Martinez, M.L., Calles, G.J., Martin, B.E.: Ontology-based Web service to recommend spare time activities. International Workshop on Information Heterogeneity and Fusion in Recommender Systems(HetRec). Barcelona, Spain (2010)

19. Minsukn, Kahng, Sagkeun, Lee, Sang-goo, Lee: Ranking in Context-Aware Recommender System. School of Computer Science and Engineering. ACM. Hyderabad, India (2011) 
20. Kavitha Devi, M.K., Thirumalai, S.R., Vinoth, K.S., Venkatesh, P.: Probabilistic Neural Network approach to Alleviate sparsity and cold start problems in Collaborative Recommender Systems. In: Proceedings of International Conference on Computational Intelligence and Computing Research (ICCIC), pp. 1-4 (2010)

21. Palmisano, C., Tuzhilin, A., Gorgoglione, M.: Using context to improve predictive modeling of customers in personalization applications. IEEE Transactions on Knowledge and Data Engineering, 20(11):1535-1549 (2008)

22. Ramirez-Garcia, X., Garcia-Valdez, M.: Restaurant Recommendations based on a Domain Model and Fuzzy Rules. In: Proceedings of International Seminary in Computer Intelligence (ISCI). Tijuana Institute of Technology. Tijuana B.C., Mexico (2012)

23. Ramzi, H.M., Baazaoui, H., Ziou, D., Ben Ghézala, H.: Towards a New Model for Context-Aware Recommendation. Departament of Infoematic, University of Sherbrooke. Québec, Canada (2012)

24. Romadhony, A., Al Faraby, S., Pudjoatmodjo, B.: Online Shopping Recommender System Using Hybrid Method. In: Proceedings of International conference of Information and Communication Technology (ICoICT) (2013)

25. Wen, Wu, Liang, He, Jing, Yang.: Evaluating Recommender Systems. Department of Computer Science and Technology. East China Normal University (2012)

26. Yanlin, Zheng, Luyi, Li, Fanglin, Zheng: Context-awareness Support for Content Recommendation in e-learning Environments. In: Proceedings of International Conference on Information Management, Innovation Management and Industrial Engineering (2009)

27. Zied, Z., Godin, R., Faucher, L.: Evaluating Recommender Systems. In: Proceedings of International Conference on Automated solutions for Cross Media Content and Multichannel Distribution. IEEE (2008)

28. Zimmermann, M., Specht, L. A.: Personalization and context management. User Modeling and User-Adapted Interaction, vol. 15, no.3-4, pp. 275-302 (2005) 